\title{
Effects of Perturbation on Formation Flights around an Eccentric Reference Orbit*
}

\author{
Katsuhiko YAMADA**, Takeya SHIMA*** and Shoji YOSHIKAWA*** \\ ** Nagoya University \\ Furo-cho, Chikusa-ku, Nagoya, Aichi 464-8603, Japan \\ *** Mitsubishi Electric Corporation \\ 8-1-1, Tsukaguchi-Honmachi, Amagasaki, Hyogo 661-8661, Japan
}

\begin{abstract}
In this study, we propose a basic method for the construction of a formation flight around an eccentric reference orbit under the influence of gravitational disturbances $\left(J_{2}\right)$. The relative motion between two spacecraft in the formation flight is affected by $J_{2}$. The effects of this perturbing force are analyzed, and the initial conditions of the formation flight for the suppression of these effects are derived on the basis of these analytical results.
\end{abstract}

Key words : Spacecraft, Formation Flight, $J_{2}$, Eccentric Orbit

\section{Nomenclature}

$\mu$ : gravity constant of the Earth

$R$ : equatorial radius of the Earth

$J_{2}$ : second-order zonal harmonic coefficient of the gravitational potential of the Earth $\left(J_{2}=1.0826 \times 10^{-3}\right)$

$r$ : position vector of the spacecraft in the inertial system

$v$ : velocity vector of the spacecraft in the inertial system

$a$ : semimajor axis of the (chief) reference orbit

$e:$ eccentricity of the reference orbit

$i$ : inclination of the reference orbit $\omega:$ argument of the perigee of the ref-

Subscripts $c$ and $d$ indicate the variables of chief spacecraft and deputy spacecraft, respectively.

\section{Introduction}

There are zonal harmonic terms in the Earth's gravity potential since the Earth's shape is not perfectly spherical. These terms are usually expressed by coefficients $J_{k}(k=2,3, \cdots)$, and the $J_{2}$ harmonic that originates from the oblateness (equator-pole radius difference) of the Earth is the dominant harmonic among them. It is well known that the $J_{2}$ harmonic has effects on the formation flight of multiple spacecraft ${ }^{(1)}$. In the case of a circular reference orbit, initial conditions that are expressed in terms of the Hill coordinates have already been derived for canceling the effects of $J_{2}{ }^{(2)}$. Even in the case of an elliptic reference orbit, the initial conditions for canceling the effects of $J_{2}$ have been derived ${ }^{(3)}$; however, the initial velocities are not expressed in terms of the time derivatives of the Hill coordinates, and thus, they are not necessarily suitable as the initial conditions for the deputy spacecraft. In this study, the initial velocity of the deputy spacecraft for canceling the effects of $J_{2}$ are derived in terms of 
the time derivative of the Hill coordinates in the case of an elliptic reference orbit.

\section{Transition matrix in Hill coordinates}

\subsection{Hill coordinates}

A spacecraft that moves on a reference orbit is called the chief spacecraft, and a spacecraft in formation flight with respect to the chief spacecraft is called the deputy spacecraft. The local orthogonal coordinate system with the origin at the center of mass of the chief spacecraft is selected so that the $x$-axis is aligned with an imaginary line drawn from the Earth's center of mass to the chief spacecraft and the $z$-axis aligns with the normal (rotation vector) to the orbital plane of the chief spacecraft. These local coordinates are called the Hill coordinates. Set the position vector of the chief spacecraft and its second derivative in terms of the inertial coordinates as $\boldsymbol{r}_{c}$ and $\ddot{\boldsymbol{r}}_{c}$, respectively. The equations of motion of the chief spacecraft are as follows:

$$
\ddot{\boldsymbol{r}}_{c}=-\frac{\mu_{e}}{r_{c}^{3}} \boldsymbol{r}_{c}+\boldsymbol{f}_{J_{2}}
$$

where $f_{J 2}$ is the force exerted by the $J_{2}$ term on the chief spacecraft. Let the components of $f_{J 2}$ be expressed in terms of the Hill coordinates as $\left[\begin{array}{lll}f_{x} & f_{y} & f_{z}\end{array}\right]^{T}$. These components are expressed as follows by using the orbit elements of the chief spacecraft ${ }^{(4)}$ :

$$
\begin{aligned}
& f_{x}=\frac{3 \mu J_{2} R^{2}}{2 r_{c}^{4}}\left(3 \sin ^{2} i_{c} \sin ^{2} \theta_{c}-1\right) \\
& f_{y}=-\frac{3 \mu J_{2} R^{2}}{2 r_{c}^{4}} \sin ^{2} i_{c} \sin 2 \theta_{c} \\
& f_{z}=-\frac{3 \mu J_{2} R^{2}}{2 r_{c}^{4}} \sin 2 i_{c} \sin \theta_{c}
\end{aligned}
$$

\subsection{Transformation of state vector}

Set the position vector $\boldsymbol{r}$ expressed in the Hill coordinates as

$$
\boldsymbol{r}=\left[\begin{array}{lll}
r_{x} & r_{y} & r_{z}
\end{array}\right]^{T}
$$

and the state vector $\boldsymbol{p}$ of the spacecraft as

$$
\boldsymbol{p}=\left[\begin{array}{llllll}
r_{x} & r_{y} & r_{z} & \dot{r}_{x} & \dot{r}_{y} & \dot{r}_{z}
\end{array}\right]^{T}
$$

The state vector of the deputy spacecraft with respect to the chief spacecraft, $\delta \boldsymbol{p}$, is given as follows:

$$
\delta \boldsymbol{p}=\boldsymbol{p}_{d}-\boldsymbol{p}_{c}
$$

Set the orbit elements of the spacecraft as

$$
\begin{aligned}
\boldsymbol{q} & =\left[\begin{array}{llllll}
a & \theta & i & q_{1} & q_{2} & \Omega
\end{array}\right]^{T} \\
\boldsymbol{s} & =\left[\begin{array}{llllll}
a & e & i & \Omega & \omega & M
\end{array}\right]^{T}
\end{aligned}
$$

and the differences between the orbit elements of the deputy spacecraft and the chief spacecraft are denoted as $\delta \boldsymbol{q}$ and $\delta \boldsymbol{s}$ :

$$
\delta \boldsymbol{q}=\boldsymbol{q}_{d}-\boldsymbol{q}_{c}, \quad \delta \boldsymbol{s}=\boldsymbol{s}_{d}-\boldsymbol{s}_{c}
$$

We first obtain the time evolution of $\delta \boldsymbol{s}$ and then obtain the relation between $\delta \boldsymbol{p}(t)$ and $\delta \boldsymbol{p}\left(t_{0}\right)$ $\left(t_{0}\right.$ : initial time) as a state transition matrix. Hereafter, we derive the relations required for this calculation. 


\subsection{Transformation between $\delta p \leftrightarrow \delta q$}

The transformation between $\delta \boldsymbol{p}$ and $\delta \boldsymbol{q}$ is expressed as follows where the partial derivatives are evaluated at the chief spacecraft state:

$$
\begin{array}{ll}
\delta \boldsymbol{p}=\boldsymbol{A}_{H q} \delta \boldsymbol{q}, & \boldsymbol{A}_{H q}=\left.\frac{\partial \boldsymbol{p}}{\partial \boldsymbol{q}}\right|_{c} \\
\delta \boldsymbol{q}=\boldsymbol{A}_{q H} \delta \boldsymbol{p}, & \boldsymbol{A}_{q H}=\boldsymbol{A}_{H q}^{-1}=\left.\frac{\partial \boldsymbol{q}}{\partial \boldsymbol{p}}\right|_{c}
\end{array}
$$

The matrices $\boldsymbol{A}_{H q}$ and $\boldsymbol{A}_{q H}$ that are under the influence of $\boldsymbol{J}_{2}$ have already been derived in the literature $^{(5)}$.

\subsection{Transformation between $\delta q \leftrightarrow \delta s$}

As $a, i$, and $\Omega$ are the common components between the orbit elements $\boldsymbol{q}$ and $\boldsymbol{s}$, the transformation between $\delta \boldsymbol{q}$ and $\delta \boldsymbol{s}$ is not a complex one. Let us set the following:

$$
\begin{array}{ll}
\delta \boldsymbol{q}=\boldsymbol{A}_{q s} \delta \boldsymbol{s}, & \boldsymbol{A}_{q s}=\left.\frac{\partial \boldsymbol{q}}{\partial \boldsymbol{s}}\right|_{c} \\
\delta \boldsymbol{s}=\boldsymbol{A}_{s q} \delta \boldsymbol{q}, & \boldsymbol{A}_{s q}=\boldsymbol{A}_{q s}^{-1}=\left.\frac{\partial \boldsymbol{s}}{\partial \boldsymbol{q}}\right|_{c}
\end{array}
$$

Since the relation between $M$ and $E$ is as follows:

$$
M=E-e \sin E,
$$

$\delta M=M_{d}-M_{c}$ around the chief spacecraft is obtained as ${ }^{(1)}$.

$$
\delta M=\frac{\sqrt{1-e_{c}^{2}}}{\left(1+e_{c} \cos v\right)^{2}}\left[\left(1-e_{c}^{2}\right) \delta v_{c}-\sin v_{c}\left(2+e_{c} \cos v_{c}\right) \delta e\right]
$$

Thus, by using $\delta v=\delta \theta-\delta \omega$, we obtain

$$
\delta \theta=\frac{\left(1+e_{c} \cos v_{c}\right)^{2}}{\left(1-e_{c}^{2}\right)^{\frac{3}{2}}} \delta M+\delta \omega+\frac{\sin v_{c}\left(2+e_{c} \cos v_{c}\right)}{1-e_{c}^{2}} \delta e_{c}
$$

Using this relation, the non-zero components of $\boldsymbol{A}_{q s}$ are obtained as follows, where the $(i, j)$ component $\boldsymbol{A}_{q s}$ is denoted as $A_{q s i j}$ :

$$
\begin{aligned}
& A_{q s 11}=A_{q s 24}=A_{q s 33}=A_{q s 64}=1 \\
& A_{q s 22}=\frac{\sin v_{c}\left(2+e_{c} \cos v_{c}\right)}{1-e_{c}^{2}} \\
& A_{q s 25}=\frac{\left(1+e_{c} \cos v_{c}\right)^{2}}{\left(1-e_{c}^{2}\right)^{\frac{3}{2}}} \\
& A_{q s 42}=\cos \omega_{c}, \quad A_{q s 45}=-e_{c} \sin \omega_{c} \\
& A_{q s 52}=\sin \omega_{c}, \quad A_{q s 55}=-e_{c} \sin \omega_{c}
\end{aligned}
$$

\subsection{Time evolution of $\delta s$}

Finally, we obtain the time evolution of $\delta s$ as a state transition matrix. We can calculate the state transition matrix in terms of the Hill coordinates by multiplying the above coefficient matrices associated with the variable transformations. By substituting $f_{x}, f_{y}$, and $f_{z}$ given by Eqs. (2)-(4) into the Gauss planetary equations and averaging them in one orbit, the variation in the mean orbit elements due to $J_{2}$ are obtained as follows ${ }^{(1)}$, where the mean orbit elements corresponding to the osculating orbit elements $a_{c}, e_{c}, i_{c}, \omega_{c}$, and $M_{c}$ are expressed as $\bar{a}_{c}, \bar{e}_{c}$, $\bar{i}_{c}, \bar{\omega}_{c}$, and $\bar{M}_{c}$, respectively:

$$
\begin{aligned}
& \frac{d \bar{a}_{c}}{d t}=0 \\
& \frac{d \bar{e}_{c}}{d t}=0
\end{aligned}
$$




$$
\begin{aligned}
\frac{d \bar{i}_{c}}{d t} & =0 \\
\frac{d \bar{\Omega}_{c}}{d t} & =-\frac{3}{2} J_{2} n_{c}\left(\frac{R}{p_{c}}\right)^{2} \cos i_{c} \\
\frac{d \bar{\omega}_{c}}{d t} & =\frac{3}{4} J_{2} n_{c}\left(\frac{R}{p_{c}}\right)^{2}\left(5 \cos ^{2} i_{c}-1\right) \\
\frac{d \bar{M}_{c}}{d t} & =\bar{n}_{c}+\frac{3}{4} J_{2} n_{c}\left(\frac{R}{p_{c}}\right)^{2} \eta_{c}\left(3 \cos ^{2} i_{c}-1\right)
\end{aligned}
$$

We can obtain the variations in the mean orbit elements using the above equation and approximate the variations in the osculating orbit elements. In Eqs. (21) and (22), the osculating orbit elements are used in their right hand sides. Because the differences between the mean orbit elements and the osculating orbit elements depend on $J_{2}$ and the second-order terms of $J_{2}$ can be neglected, both the orbit elements can be used in the right hand sides of Eqs. (21) and (22). However, $\bar{n}_{c}$, which is the mean angular motion based on $\bar{a}_{c}$, should be used in the right hand side of Eq. (23), because this term is not multiplied by $J_{2}$. In this case, by expressing $\bar{a}_{c}$ as

$$
\bar{a}_{c}=a_{c}\left(1+\Delta_{a}\right),
$$

$\bar{n}_{c}$ can be expressed as follows:

$$
\bar{n}_{c}=\sqrt{\frac{\mu}{\bar{a}_{c}^{3}}}=\sqrt{\frac{\mu}{a_{c}^{3}}}\left(1-\frac{3}{2} \Delta_{a}\right)
$$

where $\Delta a$ is calculated as ${ }^{(1)}$

$$
\begin{aligned}
\Delta_{a} & =-\frac{J_{2} R^{2}}{2 a_{c}^{2}\left(1-e_{c}^{2}\right)^{3}}\left[( 3 \operatorname { c o s } ^ { 2 } i _ { c } - 1 ) \left(\left(1+e_{c} \cos v_{c 0}\right)^{3}\right.\right. \\
& \left.\left.-\left(1-e_{c}^{2}\right)^{\frac{3}{2}}\right)+3 \sin ^{2} i_{c}\left(1+e_{c} \cos v_{c 0}\right)^{3} \cos \left(2 \omega_{c}+2 v_{c 0}\right)\right]
\end{aligned}
$$

Let us summarize Eqs. (18)-(23) as follows:

$$
\frac{d \bar{s}}{d t}=\boldsymbol{g}(\boldsymbol{s})
$$

In order to obtain the time evolution of $\delta \boldsymbol{s}$, we obtain the following approximation on the basis of the above equation.

$$
\frac{d \delta \boldsymbol{s}}{d t}=\boldsymbol{A}_{s} \delta \boldsymbol{s}, \quad \boldsymbol{A}_{s}=\left.\frac{\partial \boldsymbol{g}}{\partial \boldsymbol{s}}\right|_{c}
$$

By solving this equation, we obtain $\delta \boldsymbol{s}(t)$ as follows:

$$
\delta \boldsymbol{s}(t)=\boldsymbol{\Phi}_{s}\left(t, t_{0}\right) \delta \boldsymbol{s}\left(t_{0}\right), \quad \boldsymbol{\Phi}_{s}\left(t, t_{0}\right)=\exp \left(\boldsymbol{A}_{s}\left(t-t_{0}\right)\right)
$$

In general, it is not easy to obtain $\boldsymbol{\Phi}_{s}$; however, $\boldsymbol{\Phi}_{s}$ can be easily obtained in this case because the upper triangle part of $\boldsymbol{A}_{s}$ are zeros except for the $(6,6)$ component. Let the $(6,6)$ component of $\boldsymbol{A}_{s}$ be $a_{s 66}$, and it is given as follows:

$$
a_{s 66}=-\frac{9 J_{2} R^{2} n_{c} \sqrt{1+e_{c}} \sin \omega_{c} \cos \omega_{c} \sin ^{2} i_{c}}{a_{c}^{2}\left(1-e_{c}\right)^{\frac{9}{2}}}
$$

The time exponential function in $\boldsymbol{\Phi}_{s}\left(t, t_{0}\right)$ is $\exp \left(a_{s 66}\left(t-t_{0}\right)\right)$ only. If we consider the approximation

$$
\exp \left(a_{s 66}\left(t-t_{0}\right)\right) \approx 1+a_{s 66}\left(t-t_{0}\right),
$$

the nonzero components of $\boldsymbol{\Phi}_{s}\left(t, t_{0}\right)$ can be expressed as follows, where $\Phi_{s i j}$ and $\alpha$ represent the $(i, j)$ components of $\boldsymbol{\Phi}_{s}$ and $J_{2} R^{2} n_{c}\left(t-t_{0}\right) / a_{c}^{2}$, respectively:

$$
\Phi_{s 11}=\Phi_{s 22}=\Phi_{s 33}=\Phi_{s 44}=\Phi_{s 55}=1
$$




$$
\begin{aligned}
& \Phi_{s 41}=\frac{21 \alpha \cos i_{c}}{4 a_{c}\left(1-e_{c}^{2}\right)^{2}} \\
& \Phi_{s 42}=-\frac{6 \alpha e_{c} \cos i_{c}}{\left(1-e_{c}^{2}\right)^{3}} \\
& \Phi_{s 43}=\frac{3 \alpha \sin i_{c}}{2\left(1-e_{c}^{2}\right)^{2}} \\
& \Phi_{s 51}=-\frac{21 \alpha\left(5 \cos ^{2} i_{c}-1\right)}{8 a_{c}\left(1-e_{c}^{2}\right)^{2}} \\
& \Phi_{s 52}=\frac{3 \alpha e_{c}\left(5 \cos ^{2} i_{c}-1\right)}{\left(1-e_{c}^{2}\right)^{3}} \\
& \Phi_{s 53}=-\frac{15 \alpha \sin i_{c} \cos i_{c}}{2\left(1-e_{c}^{2}\right)^{2}} \\
& \Phi_{s 61}=-\frac{3 n_{c}\left(t-t_{0}\right)}{2 a_{c}}+\frac{21 \alpha\left(3 \sin ^{2} \omega_{c} \sin ^{2} i_{c}-1\right)}{4 a_{c}\left(1-e_{c}\right)^{3}} \\
& \Phi_{s 62}=-\frac{9 \alpha\left(3 \sin ^{2} \omega_{c} \sin ^{2} i_{c}-1\right)}{2\left(1-e_{c}\right)^{4}} \\
& \Phi_{s 63}=-\frac{9 \alpha \sin ^{2} \omega_{c} \sin i_{c} \cos i_{c}}{\left(1-e_{c}\right)^{3}} \\
& \Phi_{s 65}=-\frac{9 \alpha \sin _{c} \omega_{c} \cos \omega_{c} \sin ^{2} i_{c}}{\left(1-e_{c}\right)^{3}} \\
& \Phi_{s 66}=1-\frac{9 \alpha \sqrt{1+e_{c}} \sin _{c} \omega_{c} \cos _{c} \omega^{2} \sin ^{2} i_{c}}{\left(1-e_{c}\right)^{\frac{9}{2}}}
\end{aligned}
$$

\subsection{Time evolution of $\delta p$}

Using the abovementioned procedure, the state transition matrix between $\delta \boldsymbol{p}(t)$ and $\delta \boldsymbol{p}\left(t_{0}\right)$ can be obtained. Let the state transition matrix be

$$
\delta \boldsymbol{p}(t)=\boldsymbol{\Phi}_{p}\left(t, t_{0}\right) \delta \boldsymbol{p}\left(t_{0}\right),
$$

where $\boldsymbol{\Phi}_{p}\left(t, t_{0}\right)$ is expressed as follows:

$$
\boldsymbol{\Phi}_{p}\left(t, t_{0}\right)=\boldsymbol{A}_{H q}(t) \boldsymbol{A}_{q s}(t) \boldsymbol{\Phi}_{s}\left(t, t_{0}\right) \boldsymbol{A}_{s q}\left(t_{0}\right) \boldsymbol{A}_{q H}\left(t_{0}\right)
$$

It should be noted that the components of $\boldsymbol{A}_{H q}, \boldsymbol{A}_{q s}, \boldsymbol{\Phi}_{s}, \boldsymbol{A}_{s q}$, and $\boldsymbol{A}_{q H}$ are the functions of the orbit elements of the chief spacecraft: $a_{c}, e_{c}, i_{c}, \omega_{c}$, and $v_{c}$, respectively. Therefore, the orbit elements at time $t_{0}$ are used to obtain $\boldsymbol{\Phi}_{s}\left(t, t_{0}\right), \boldsymbol{A}_{s q}\left(t_{0}\right)$ and $\boldsymbol{A}_{q H}\left(t_{0}\right)$ and those at time $t$ are used to obtain $\boldsymbol{A}_{H q}(t)$ and $\boldsymbol{A}_{q s}(t)$. However, as the time variations in the mean orbit elements $a_{c}, e_{c}$, and $i_{c}$ can be neglected under the influence of $J_{2}$, the values of these elements can be considered to be the same at $t_{0}$ and $t$. The true anomaly $v_{c}$ is used to designate time as well as $t$. The mean orbit element of the argument of the perigee $\omega_{c}$ varies as shown in Eq. (22) and it is approximated as

$$
\omega_{c}(t)=\omega_{c}\left(t_{0}\right)+\beta, \quad \beta=\frac{3 \alpha\left(5 \cos ^{2} i_{c}-1\right)}{4\left(1-e_{c}^{2}\right)^{2}}
$$

Then, $\sin \left(\omega_{c}(t)\right)$ and $\cos \left(\omega_{c}(t)\right)$ are approximated as follows:

$$
\begin{aligned}
& \sin \left(\omega_{c}(t)\right) \approx \sin \left(\omega_{c}\left(t_{0}\right)\right)+\cos \left(\omega_{c}\left(t_{0}\right)\right) \beta \\
& \cos \left(\omega_{c}(t)\right) \approx \cos \left(\omega_{c}\left(t_{0}\right)\right)-\sin \left(\omega_{c}\left(t_{0}\right)\right) \beta
\end{aligned}
$$

We set the time at the perigee as the initial time $t_{0}$ and the true anomaly of the chief spacecraft at time $t$ as $v_{c}$. We also set each component of $\delta \boldsymbol{p}$ at time $t$ in terms of the Hill coordinates as

$$
\delta \boldsymbol{p}=\left[\begin{array}{llllll}
p_{x} & p_{y} & p_{z} & v_{x} & v_{y} & v_{z}
\end{array}\right]^{T}
$$


where $v_{x}=\dot{p}_{x}, v_{y}=\dot{p}_{y}$, and $v_{z}=\dot{p}_{z}$. By using the subscript 0 to represent the variables at time $t_{0}, p_{x}, p_{y}$, and $p_{z}$ are calculated by using $\boldsymbol{\Phi}_{p}\left(t, t_{0}\right)$ from $p_{x 0}, p_{y 0}$ and $p_{z 0}$, respectively. When the effects of $J_{2}$ are ignored, the following equations should be satisfied in order to cancel the time-dependent terms of $p_{x}$ and $p_{y}$ :

$$
v_{y 0}=\frac{\left(2+e_{c}\right) n_{c}}{\sqrt{\left(1+e_{c}\right)\left(1-e_{c}\right)^{3}}} p_{x 0}
$$

This equation is known as the formation condition for an elliptic reference orbit ${ }^{(1)}$. Let us express $v_{y 0}$ as follows by using this equation:

$$
v_{y 0}=\frac{\left(2+e_{c}\right) n_{c}}{\sqrt{\left(1+e_{c}\right)\left(1-e_{c}\right)^{3}}} p_{x 0}+\Delta v_{y 0}
$$

Then, $p_{x}$ and $p_{y}$ are expressed as follows:

$$
\begin{aligned}
p_{x} & =\cos v_{c} p_{x 0}+\frac{\left(1-e_{c}\right)^{\frac{3}{2}} \sin v_{c}}{n_{c} \sqrt{1+e_{c}}} v_{x 0} \\
& +\left[\frac{2 \sqrt{1-e_{c}^{2}}\left(1-\cos v_{c}\right)\left(1+e_{c}+e_{c} \cos v_{c}\right)}{n_{c}\left(1+e_{c} \cos v_{c}\right)}-\frac{3 e_{c} \sin v_{c}\left(t-t_{0}\right)}{1-e_{c}}\right] \Delta v_{y 0} \\
p_{y} & =\frac{\left(2+e_{c} \cos v_{c}\right) \sin v_{c}}{1+e_{c} \cos v_{c}} p_{x 0}+\frac{1+e_{c}}{1+e_{c} \cos v_{c}} p_{y 0} \\
& -\frac{\left(1-e_{c}\right)^{\frac{3}{2}}\left(2+e_{c}+e_{c} \cos v_{c}\right)\left(1-\cos v_{c}\right)}{n_{c} \sqrt{1+e_{c}}\left(1+e_{c} \cos v_{c}\right)} v_{x 0} \\
& +\left[\frac{2 \sqrt{1-e_{c}^{2}}\left(2+e_{c} \cos v_{c}\right) \sin v_{c}}{n_{c}\left(1+e_{c} \cos v_{c}\right)}-\frac{3\left(1+e_{c} \cos v_{c}\right)\left(t-t_{0}\right)}{1-e_{c}}\right] \Delta v_{y 0} \\
p_{z}= & \frac{\left(1+e_{c}\right) \cos v_{c}}{1+e_{c} \cos v_{c}} p_{z 0}+\frac{\sqrt{1+e_{c}}\left(1-e_{c}\right)^{\frac{3}{2}} \sin v_{c}}{n_{c}\left(1+e_{c} \cos v_{c}\right)} v_{z 0}
\end{aligned}
$$

As described later, there are time-dependent terms in $p_{x}, p_{y}$, and $p_{z}$ due to the influence of $J_{2}$. Some of these time-dependent terms can be cancelled by using the time-dependent terms obtained using $\Delta v_{y 0}$. This procedure is described in the next section.

\section{Initial conditions considering the effects of $J_{2}$}

\subsection{Formation condition}

When the chief spacecraft and deputy spacecraft are in formation flight, there is a gradual change in the formation flight due to the effects of $J_{2}$. In this study, we consider the initial conditions to minimize the changes in formation due to the effects of $J_{2}$. Hereafter, by considering $\delta \boldsymbol{p}$ as a function of $v_{c}$, we set $\delta \boldsymbol{p}$ at the perigee as follows:

$$
\delta \boldsymbol{p}\left(v_{c}=0\right)=\left[\begin{array}{llllll}
p_{x 0} & p_{y 0} & p_{z 0} & v_{x 0} & v_{y 0} & v_{z 0}
\end{array}\right]^{T}
$$

For this initial condition, we set $\delta \boldsymbol{p}$ at $v_{c}=v_{c f}$ as follows:

$$
\delta \boldsymbol{p}\left(v_{c}=v_{c f}\right)=\left[\begin{array}{llllll}
p_{x f} & p_{y f} & p_{z f} & v_{x f} & v_{y f} & v_{z f}
\end{array}\right]^{T}
$$

In the following two cases, the effects of $J_{2}$ on the formation flight are analyzed:

$$
\begin{array}{lll}
\text { Case } 1: & p_{y f}=p_{y 0} \text { at } v_{c f}=2 N \pi \quad(N \text { : integer }) \\
\text { Case } 2: & \frac{d}{d t}(\delta M+\delta \omega)=0
\end{array}
$$

In Case 1, the deputy spacecraft returns to the initial position in the Hill coordinates after orbiting $N$ times, where $N$ is assumed to be in the range of $\beta \ll 1$. In Case 2 , the deviations in the orbit elements $M+\omega$ of the deputy spacecraft from those of the chief spacecraft do not change with time. 


\subsection{Case 1}

Set the time at $v_{c}=2 N \pi$ as $t_{f}$. When $v_{y 0}$ is set as shown in Eq. (37), $p_{x f}, p_{y f}$, and $p_{z f}$ are obtained in the following form with the inclusion of the effects of $J_{2}$ :

$$
\begin{aligned}
& p_{x f}=p_{x 0} \\
& p_{y f}=p_{y 0}+p_{y J 2}-\frac{3\left(1+e_{c}\right)\left(t_{f}-t_{0}\right)}{1-e_{c}} \Delta v_{y 0} \\
& p_{z f}=p_{z 0}+p_{z J 2}
\end{aligned}
$$

where $p_{y J 2}$ and $p_{z J 2}$ are the terms that are introduced due to the effects of $J_{2}$. As shown in Eq. (43), the effects of $J_{2}$ do not appear in $p_{x f}$ in the first order. Therefore, $\Delta v_{y 0}$ is determined from the relation $p_{y f}=p_{y 0}$ :

$$
\Delta v_{y 0}=\frac{1-e_{c}}{3\left(1+e_{c}\right)\left(t_{f}-t_{0}\right)} p_{y J 2}
$$

In this case, $p_{z J 2}$ is obtained as follows:

$$
\begin{aligned}
p_{z J 2} & =-\frac{3 J_{2} R^{2}\left(t_{f}-t_{0}\right) \cos \omega_{c} \sin i_{c}}{a_{c}^{2}\left(1+e_{c}\right)^{3}\left(1-e_{c}\right)^{2}}\left(2 n_{c} e_{c} \cos i_{c} p_{x 0}+n_{c}\left(1+e_{c}\right) \sin \omega_{c} \cos i_{c} p_{z 0}\right. \\
& \left.+\frac{1}{4} \sqrt{1+e_{c}}\left(1-e_{c}\right)^{\frac{3}{2}}\left(1+2 \cos ^{2} \omega_{c} \sin ^{2} i_{c}-5 \cos ^{2} i_{c}\right) v_{z 0}\right)
\end{aligned}
$$

Although $p_{z J 2}$ cannot be cancelled by $\Delta v_{y 0}$, the effects of $p_{z J 2}$ is relatively small as compared with those of $p_{y J 2}$. This is because the initial condition of $p_{y 0}$ (in the direction of motion) does not affect $p_{z J 2}$ and the effects of the initial condition of $v_{z 0}$ (in the out-of-plane direction) are cancelled by a small phase difference in the vicinity of $v_{c}=2 N \pi$, as shown in Eq. (40). $\Delta v_{y 0}$ is obtained using Eq. (46) as follows, where the orbit elements of the chief spacecraft are the initial values:

$$
\begin{aligned}
v_{y 0} & =\left[-\frac{n_{c}\left(2+e_{c}\right)}{\sqrt{\left(1+e_{c}\right)\left(1-e_{c}\right)^{3}}}+\frac{3 J_{2} R^{2} n_{c}\left(3 \sin ^{2} \omega_{c} \sin ^{2} i_{c}-1\right)}{2 a_{c}^{2} \sqrt{1+e_{c}}\left(1-e_{c}\right)^{\frac{7}{2}}}-\frac{J_{2} R^{2} n_{c} e_{c}\left(3 \cos ^{2} i_{c}-1\right)}{a_{c}^{2}\left(1+e_{c}\right)^{4}\left(1-e_{c}\right)}\right] p_{x 0} \\
& -\frac{3 J_{2} R^{2} n_{c} \sin 2 \omega_{c} \sin ^{2} i_{c}}{2 a_{c}^{2} \sqrt{1+e_{c}}\left(1-e_{c}\right)^{\frac{7}{2}}} p_{y 0}-\left[\frac{3 J_{2} R^{2} n_{c} \sin \omega_{c} \sin 2 i_{c}}{a_{c}^{2} \sqrt{1+e_{c}}\left(1-e_{c}\right)^{\frac{7}{2}}}+\frac{J_{2} R^{2} n_{c} \sin \omega_{c} \sin 2 i_{c}}{a_{c}^{2}\left(1+e_{c}\right)^{3}\left(1-e_{c}\right)}\right] p_{z 0} \\
& -\frac{J_{2} R^{2} \sqrt{1-e_{c}} \cos \omega_{c} \sin 2 i_{c}}{a_{c}^{2}\left(1+e_{c}\right)^{\frac{7}{2}}} v_{z 0}
\end{aligned}
$$

\subsection{Case 2}

Since the condition of Case 2 is obtained from the deviation of the orbit elements, it is evaluated as follows from the time evolution of $\delta \boldsymbol{s}$ in Eq. (27):

$$
\frac{d \delta \boldsymbol{s}}{d t}=\boldsymbol{A}_{s}\left(t_{0}\right) \boldsymbol{A}_{s q}\left(t_{0}\right) \boldsymbol{A}_{q H}\left(t_{0}\right) \delta \boldsymbol{p}\left(t_{0}\right)
$$

When $v_{y 0}$ is given in Eq. (37) in the initial condition of $\delta \boldsymbol{p}\left(t_{0}\right)$, the term

$$
\frac{d}{d t}(\delta \omega+\delta M)
$$

can be evaluated by adding the fifth row and the sixth row of Eq. (49). When the value of the above term is 0 , the effects of $J_{2}$ are decreased in the orbital plane. $v_{y 0}$ is derived from this condition and the result is obtained as follows:

$$
\begin{aligned}
v_{y 0} & =\left[-\frac{n_{c}\left(2+e_{c}\right)}{\sqrt{\left(1+e_{c}\right)\left(1-e_{c}\right)^{3}}}+\frac{3 J_{2} R^{2} n_{c}\left(3 \sin ^{2} \omega_{c} \sin ^{2} i_{c}-1\right)}{2 a_{c}^{2} \sqrt{1+e_{c}}\left(1-e_{c}\right)^{\frac{7}{2}}}-\frac{J_{2} R^{2} n_{c} e_{c}\left(5 \cos ^{2} i_{c}-1\right)}{a_{c}^{2}\left(1+e_{c}\right)^{\frac{7}{2}}\left(1-e_{c}\right)^{\frac{5}{2}}}\right] p_{x 0} \\
& -\frac{3 J_{2} R^{2} n_{c} \sin 2 \omega_{c} \sin ^{2} i_{c}}{2 a_{c}^{2} \sqrt{1+e_{c}}\left(1-e_{c}\right)^{\frac{7}{2}}} p_{y 0}-\left[\frac{3 J_{2} R^{2} n_{c} \sin \omega_{c} \sin 2 i_{c}}{a_{c}^{2} \sqrt{1+e_{c}}\left(1-e_{c}\right)^{\frac{7}{2}}}+\frac{5 J_{2} R^{2} n_{c} \sin \omega_{c} \sin 2 i_{c}}{4 a_{c}^{2}\left(1+e_{c}\right)^{\frac{5}{2}}\left(1-e_{c}\right)^{\frac{5}{2}}}\right] p_{z 0} \\
& -\frac{5 J_{2} R^{2} \cos \omega_{c} \sin 2 i_{c}}{4 a_{c}^{2}\left(1+e_{c}\right)^{3}\left(1-e_{c}\right)} v_{z 0}
\end{aligned}
$$

This result is slightly different from Eq. (48). 


\section{Numerical simulation results}

\subsection{Initial conditions of an elliptic orbit}

By focusing on the case where the chief spacecraft moves on an elliptic orbit, we set the initial conditions of the deputy spacecraft in terms of the Hill coordinates as follows:

$$
\begin{array}{ll}
p_{x 0}=0, \quad p_{y 0}=l, & p_{z 0}=0 \\
v_{x 0}=\frac{n_{c} \sqrt{1+e_{c}} l}{2\left(1-e_{c}\right)^{\frac{3}{2}}}, & v_{y 0}=0, \quad v_{z 0}=\frac{n_{c} l}{1-e_{c}}
\end{array}
$$

These initial conditions are set so that a nearly circular formation is realized in the case of Keplerian motion without any disturbances ${ }^{(6)}$. Simulation results are shown in Figs. 1 and 2 when the orbit elements of the chief spacecraft at the initial time are set as

$$
\begin{array}{ll}
e_{c}=0.5, \quad l=1[\mathrm{~km}], & \Omega_{c}=0 \\
\omega_{c}=60^{\circ}, \quad i_{c}=30^{\circ}, & a_{c}=\frac{R+500[\mathrm{~km}]}{1-e_{c}}
\end{array}
$$

Figure 1 shows the case without the effects of $J_{2}$, while Fig. 2 shows the case with the effects of $J_{2}$. As shown in these figures, the formation trajectory of the deputy spacecraft is gradually disturbed by the effects of $J_{2}$.

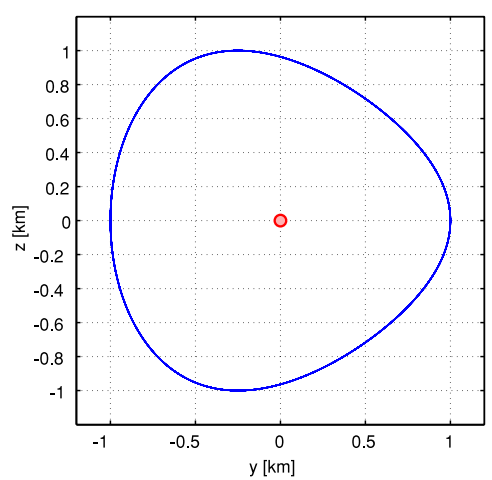

(a) trajectory in $p_{x} p_{y}$-plane

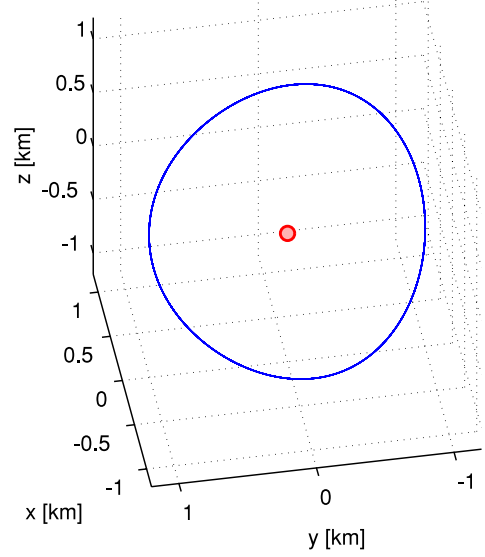

(b) trajectory in $p_{x} p_{y} p_{z}$-space

Fig. 1 Nominal deputy motion expressed in the Hill Coordinates (without the effects of $J_{2}$ )

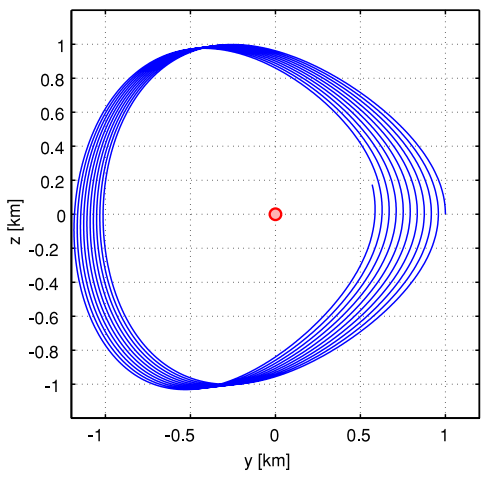

(a) trajectory in $p_{x} p_{y}$-plane

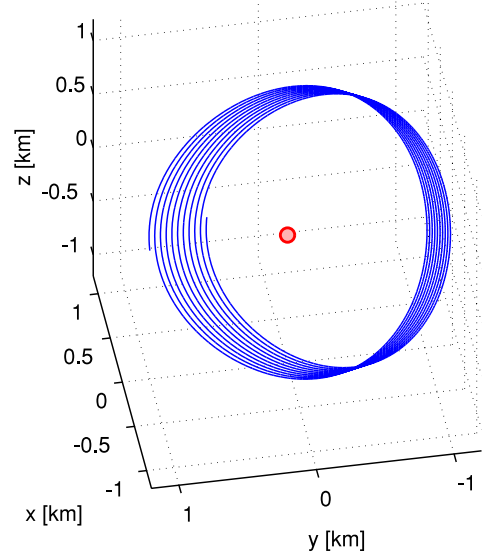

(b) trajectory in $p_{x} p_{y} p_{z}$-space

Fig. 2 Nominal deputy motion expressed in the Hill coordinates (with the effects of $J_{2}$ ) 


\subsection{Correction of initial conditions}

Next, when the effects of $J_{2}$ are present, the simulation results with the correction of the initial conditions are shown. Figure 3 shows the simulation results with the correction of Eq. (48) for Case 1, and Fig. 4 shows the simulation results with the correction of Eq. (50) for Case 2. As is clear from these results, the drift in the $y$ component can be suppressed by the correction of the initial conditions. In Case 1, the deputy spacecraft almost returns to the initial position in the Hill coordinates at the perigee. In Case 2, although there is a very small difference between the position of the deputy spacecraft at the perigee and its initial position in the Hill coordinates, the whole variation of the relative position is also suppressed.

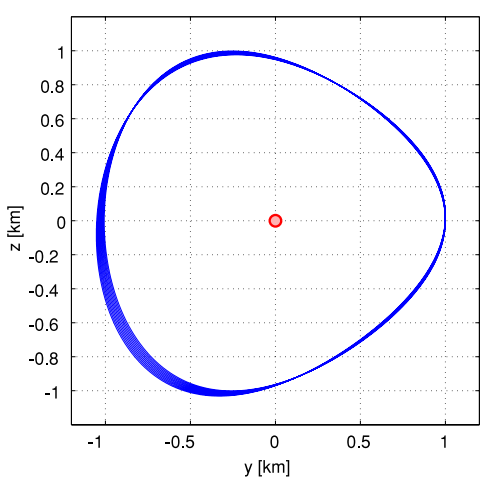

(a) trajectory in $p_{x} p_{y}$-plane

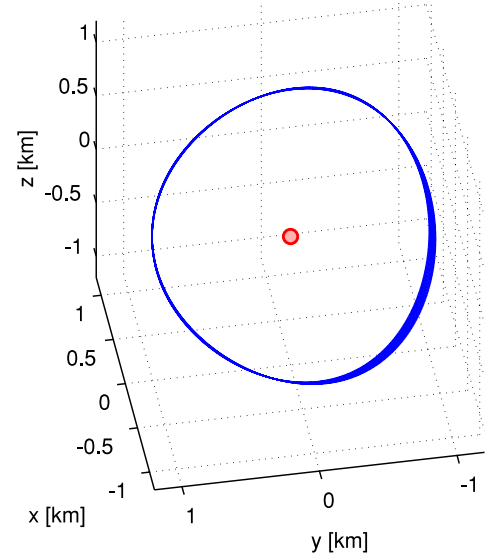

(b) trajectory in $p_{x} p_{y} p_{z}$-space

Fig. 3 Deputy motion expressed in the Hill coordinates (with $v_{y 0}$ correction in Case 1)

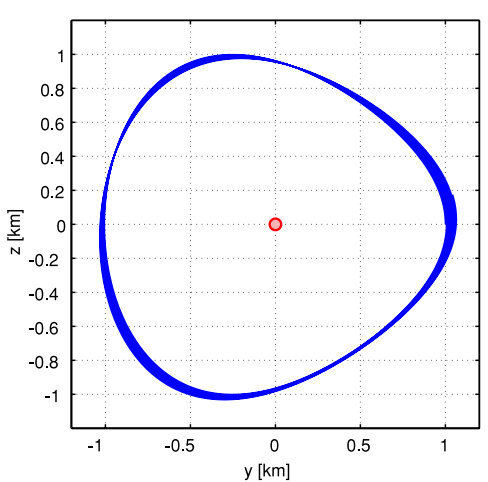

(a) trajectory in $p_{x} p_{y}$-plane

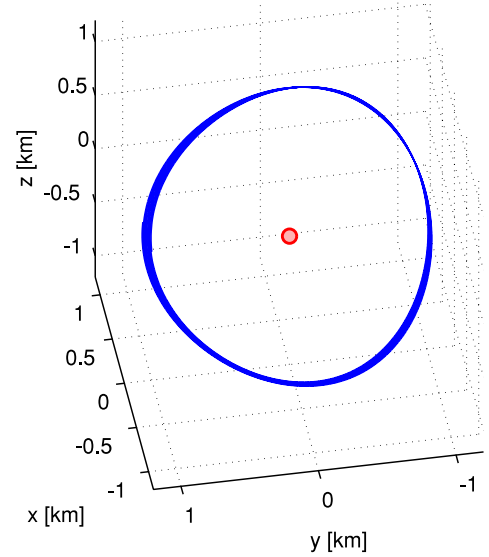

(b) trajectory in $p_{x} p_{y} p_{z}$-space

Fig. 4 Deputy motion expressed in the Hill coordinates (with $v_{y 0}$ correction in Case 2)

\section{Conclusions}

In this study, we have analyzed the effects of $J_{2}$ on the formation flight in an elliptic reference orbit and have derived the initial velocity in the direction of motion of the deputy spacecraft for canceling the effects of $J_{2}$. We have used two methods: one is to derive the state transition matrix in terms of the Hill coordinates and to obtain the initial velocity on basis of the transition matrix in order to cancel the changes in the position after orbiting $N$ times. The other one is to derive the initial velocity which makes the time change of the difference of the orbit elements between two spacecraft zero. The effectiveness of the initial velocities in canceling the effects of $J_{2}$ is verified by numerical simulations. 
The method of this paper is a kind of feedforward adjustment of the relative velocity of the deputy spacecraft in the along-track direction for canceling the effects of $J_{2}$. The $J_{2}$ effects are actually observed in the variation of the relative distance between two spacecraft at every orbit period. Therefore, a kind of feedback compensation of the $J_{2}$ effects is possible when the relative velocity of the deputy spacecraft is set in the same way as above at every orbit period based on the observation of the relative distance.

\section{References}

( 1 ) Schaub, S. and Junkins, J.L., Analytical Mechanics of Space Systems, AIAA Educational Series, pp. 489-543, 593-628, 693-696, 2003

( 2 ) Shima, T., Yamada, K., Jikuya, I. and Yoshikawa, S., Trajectory Planning of a Spacecraft based on the Modified Hill's Equation, Transactions of the Japan Society of Mechanical Engineers, Vol. 72, No. 722, pp. 3364-3371, 2006 (in Japanese)

( 3 ) Sengupta, P., Sharma, R. and Vadali, S.R., Periodic Relative Motion Near a Keplerian Elliptic Orbit with Nonlinear Differential Gravity, Journal of Guidance, Control, and Dynamics, Vol. 29, No. 5, pp. 1110-1121, 2006

( 4 ) Wie, B., Space Vehicle Dynamics and Control, AIAA Educational Series, pp. 233-240, 1998

( 5 ) Gim, D. and Alfriend, K.T., State Transition Matrix of Relative Motion for the Perturbed Noncircular Reference Orbit, Journal of Guidance, Control, and Dynamics, Vol. 26, No. 6, pp. 956-971, 2003

( 6 ) Yamada, K., Shima, T. and Yoshikawa, S., Spacecraft Formation Flying in Eccentric Orbits, Proceedings of 16th Workshop on JAXA Astrodynamics and Flight Mechanics, pp. 22-27, 2006 